In Wayne Gray and Christian Schunn, Eds., Proceedings of the Twenty-Fourth Annual Conference of the Cognitive ScienceSociety. Mahwah, NJ: Erlbaum,2002.

\title{
Protocol Evidence On Thought Experiments Used By Experts
}

\author{
John J. Clement (clement@srri.umass.edu) \\ Scientific Reasoning Research Institute College of \\ Natural Sciences and Mathematics and School of \\ Education Lederle GRT 434 University of \\ Massachusetts Amherst, MA 01003 USA
}

Despite recent advances, the Fundamental Paradox of Thought Experiments continues to challenge us: How can findings that carry conviction result from a new experiment conducted entirely within the head?" The data base for this study comes from ten professors and advanced graduate students in scientific fields who were recorded while thinking aloud about the following spring problem:

A weight is hung on a spring. The original spring is replaced with a spring made of the same kind of wire, with the same number of coils, but with coils that are twice as wide in diameter. Will the spring stretch from its natural length more, less, or the same amount under the same weight? (Assume the mass of the spring is negligible.) Why do you think so?

Clement (1989) documented analogies, Aha! insights and cyclical model evaluation and revision processes in these protocols. Working from these transcripts, a variety of untested thought experiments (in the broad sense) have also been identified, characterized as the act of making a prediction for an untested, concrete, but absent situation (the "experiment"). Aspects of the experiment must be new and untested in the sense that the subject is not informed about its behavior. In a case study of one subject, S2, whether the spring wire is bending or twisting eventually becomes a central issue. Textbooks tell us that it is twisting, whereas many subjects assume bending. S2 examined what the effect of twisting would be in the following Elemental untested thought experiment used to make a prediction for the base of an analogy to short and long rods being twisted:

(1) If I have a longer (raises hands apart over table) rod and $I$ put a twist on it (moves right hand as if twisting something), it seems to me--again, physical intuition-that it will twist more...I think I trust that intuition. I'm imagining holding something that has a certain twistyness to it, a-and twisting it. Now I'm confirming (moves right hand close to left hand,) that... as (repeats motion) I bring my hand up closer and closer to the original place where I hold it, I realize very clearly that it will get harder and harder to twist.

Bold type above identifies examples of (both kinesthetic and other) imagery-related observation categories: personal action projections, depictive hand motions, and dynamic imagery reports, in that order. None are infallible indicators on their own, but together they are most plausibly explained using a framework that includes flexible perceptual motor schemas that generate and run imagistic simulations, via the extended application of a schema outside of its normal domain, implicit knowledge, or spatial reasoning (Clement, 1994). One can point to such sources as potential origins of conviction in TEs, to help us begin to explain the fundamental paradox. They can also explain the effectiveness of the extreme case at the end of the transcript above as an example of "imagery enhancement," a phenomenon difficult to explain in other ways (ibid.).

A second concept of TE in a narrower sense that I have found useful is what I call an evaluative Gedanken experiment: This is a special kind of untested TE designed or selected by the subject to help evaluate a concept, model or theory. An example is the case of a spring made of a vertically oriented band of material (the reader might imagine the metal unwound from a coffee can, reshaped to make a spring, say, 3" wide.) The subject imagined that such a spring would still be quite stretchable even though it "cannot bend in the up-down direction," challenging the necessity of bending as "not particularly relevant at all." In this type of evaluatory Gedanken experiment he designs a special case where the bending model yields a prediction, (no stretch) but where he also has some other independent source of information that can evaluate that prediction.

I believe both the broad and narrow concepts of TEs as clarified here are useful, and both can be analyzed in think aloud protocols. The broad concept is appropriate for expressing the fundamental paradox. The narrower concept of an evaluatory Gedanken experiment encompasses some famous TEs in the history of science, impressive in that they can even contribute to eliminating an established theory.

\section{Acknowledgements}

The research reported in this study was supported by the National Science foundation under Grant RED-9453084.

\section{References}

Clement, J. (1994). Use of physical intuition and imagistic simulation in expert problem solving. Tirosh, D. (Eds.), Implicit and explicit knowledge. Norwood, NJ: Ablex Publishing Corp.

Clement, J. (1989). Learning via model construction and criticism: Protocol evidence on sources of creativity in science. Glover, J., Ronning, R., and Reynolds, C. (Eds.), Handbook of creativity: Assessment, theory and research. NY: Plenum. 